\title{
Independent associations of dairy and calcium intakes with colorectal cancers in the Adventist Health Study-2 cohort
}

\author{
Yessenia Tantamango-Bartley, Synnove F Knutsen, Karen Jaceldo-Siegl, Jing Fan, \\ Andrew Mashchak and Gary E Fraser* \\ Adventist Health Study, Loma Linda University, 24951 North Circle Drive, NH 2031, Loma Linda, CA 92350, USA
}

Submitted 10 September 2016: Final revision received 25 April 2017: Accepted 25 May 2017: First published online 24 July 2017

\begin{abstract}
Objective: Results associating dairy and Ca intakes with colorectal cancer (CRC) risk have been mixed. Most previous analyses have suffered from confounding between dairy and $\mathrm{Ca}$ intakes. We examined independent associations between these variables, also dairy foods, and CRC incidence in a population with a large range of dairy intakes.

Design: Adventist Health Study-2 is a cohort study where subjects were enrolled 2002-2007. Proportional hazard regression analyses were performed to estimate hazard ratios (HR). Regression calibration was used to correct for dietary measurement error.

Setting: The population lived in all states of the USA.

Subjects: There were 77712 analytic subjects, all of whom were Seventh-day Adventists. Much of their dietary Ca came from non-dairy sources.

Subjects: During a mean follow-up of $7 \cdot 8$ years, 380 incident colon cancer and 111 incident rectal cancer cases were observed.

Results: Comparing extreme quintiles of intake in measurement error-corrected analyses, dairy intake ( $\mathrm{HR}=0.31 ; 95 \% \mathrm{CI} 0.09,0.88)$, independent of total Ca, was inversely related with risk of rectal cancer but gave little indication of association with colon cancer. However, total Ca intake (independent of dairy) was associated with risk of colon cancer ( $\mathrm{HR}=0.55$; $95 \% \mathrm{CI} 0 \cdot 28,0 \cdot 98)$ and there was little indication of association with rectal cancer. Traditional regression analyses and associations with macronutrients from dairy generally supported these results. Milk intake was also negatively associated with CRC ( $\mathrm{HR}=0 \cdot 63 ; 95 \%$ CI $0 \cdot 43,0 \cdot 89$ ).

Conclusions: Dairy intake may decrease the risk of rectal cancer, and Ca may reduce risk of colon cancer and CRC.
\end{abstract}

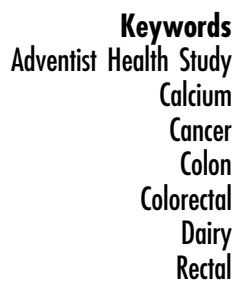

Colorectal cancer (CRC) is the third and second most common cancer in men and women respectively, worldwide. Almost $55 \%$ of cases occur in more developed countries $^{(1)}$ and CRC is the third most common cancer in both genders in the USA ${ }^{(2)}$.

Results from international correlation and migrant studies suggest that environmental factors, including diet, may cause many cases of $\mathrm{CRC}^{(3)}$. Observational studies $^{(4-14)}$ find that higher intakes of red meat and fat from animal sources (or saturated fat), and lower intakes of vegetables, fruits, $\mathrm{Ca}$ and dietary fibre, are associated with increased risk of CRC.

Dairy foods are consumed frequently around the world. The National Health and Nutrition Examination Survey estimated that $92 \%$ of Americans consume dairy products. Dairy products and Ca may affect health, including the risk of cancer. Intriguingly, they may have both protective and harmful effects ${ }^{(15)}$. For CRC, many epidemiological studies have suggested protection, but as yet results are inconclusive $^{(16-19)}$. The World Cancer Research Fund/American Institute for Cancer Research Continuing Update Report ${ }^{(20)}$ classifies dairy and $\mathrm{Ca}$ as 'probable' protective agents for CRC.

Preliminary investigations from the Adventist Health Study-2 population found a reduced incidence of cancers of the gastrointestinal tract among those who adopted a lacto-ovo-vegetarian dietary pattern ${ }^{(21)}$. The range of dairy product consumption in this cohort is wide, as some are vegan (no dairy), many are lacto-ovo-vegetarian (low dairy on average) and about half are non-vegetarian (typical dairy consumption). Moreover, as much of their $\mathrm{Ca}$ intake comes from vegetable sources, the correlation 
between $\log (\mathrm{Ca})$ and $\log$ (dairy food) intakes, as used in analyses, was only 0.35 . Thus, variance inflation and collinearity are not major problems when evaluating independent associations of these variables. Within this unusually informative population, in the present study we investigated independent associations between dairy foods, total dairy intake, as well as Ca from different sources (including supplements), and the risk of CRC.

\section{Methods}

\section{Population}

The Adventist Health Study-2 is an ongoing prospective study designed to investigate the association between diet and lifestyle factors and various diseases including cancer. A detailed description of the study design has been published $^{(22)}$. The study was conducted according to the guidelines laid down in the Declaration of Helsinki and all procedures involving human subjects/patients were approved by the Loma Linda University Internal Review Board. Written informed consent was obtained from all subjects/patients.

Enrolment between 2002 and 2007 was indicated by completion of a detailed baseline questionnaire that included the food frequency items. Finally, there were 96001 enrolees to the present study, all US or Canadian members of the Adventist Health Study-2. Of these approximately $25 \%$ were Black subjects (African Americans and West Indians living in the USA or Canada). Other races were labelled 'White' and included about 3\% Hispanics and $3 \%$ Asians. In total, individuals included in the present study represented an estimated $25-30 \%$ of the American Adventist population eligible to participate.

Linkage with cancer registries was possible for the 90844 Adventist Health Study-2 participants who lived in forty-eight US states or Washington DC. Excluded were those with: (i) age less than 25 years or missing data for age or sex ( $n$ 446); (ii) improbable response patterns in questionnaire data ( $n$ 369); (iii) greater than sixty-nine missing values in dietary data ( $n$ 1720); (iv) estimated energy intake $<2093 \mathrm{~kJ} / \mathrm{d}(<500 \mathrm{kcal} / \mathrm{d})$ or $>18828 \mathrm{~kJ} / \mathrm{d}$ ( $>4500 \mathrm{kcal} / \mathrm{d} ; n$ 3174); (v) a history of prevalent cancers ( $n$ 7402), (vi) no informed consent ( $n$ 17); and (vii) missing date of cancer diagnosis ( $n$ 4). Therefore, the final analytic population comprised 77712 subjects.

\section{Data collection and exposures of interest}

At baseline, study participants completed a validated selfadministered mailed $\mathrm{FFQ}^{(23)}$, which assessed the usual diet during the past year. This questionnaire contained over 200 food items including fruits, vegetables, legumes, grains, oils, dairy products, meats, beverages, and commercially prepared products such as dietary supplements, dry cereals, meat substitutes and soya products. Participants reported their average frequency of intake and usual serving size using predefined frequency and serving size categories according to the food under evaluation.

One variable of interest for the current study was total dairy ${ }^{(24)}$. Total kilocalories (i.e. energy from) and grams of dairy intake, and grams of intake of individual dairy foods (cheese, milk and yoghurt), were computed by multiplying the frequency of consumption of each dairy product by the kilocalories or grams of dairy content of the specific serving size. We prefer units of $\mathrm{kcal} / \mathrm{d}$ over $\mathrm{g} / \mathrm{d}$ for total dairy, given that the latter has an over-weighting for milk consumption, given its content of water, that is presumably biologically inert in this context. Nevertheless, an analysis for total dairy using $\mathrm{g} / \mathrm{d}$ is included (Table 4) for comparison with other studies that used this metric. Fat, protein and carbohydrate from dairy products were also assessed in the present study. Detailed descriptions of the methods for measuring nutrient intake using the FFQ, and its validation by repeated $24 \mathrm{~h}$ recalls, have been published elsewhere ${ }^{(25)}$. Dairy protein had an energyadjusted de-attenuated validity correlation of 0.77 (95\% CI $0.73,0.80)$ for Whites and 0.58 (95\% CI 0.51, 0.64) for Blacks. For dairy fat, the corresponding correlations were 0.66 (95\% CI $0.61,0.71$ ) and 0.56 (95\% CI $0.49,0.62$ ), respectively ${ }^{(25)}$.

$\mathrm{Ca}$ was also of special interest, specifically total $\mathrm{Ca}, \mathrm{Ca}$ from dietary sources, from dairy products and from supplements. Energy-adjusted validity correlations were 0.63 (95\% CI 0.58, 0.68) for White and 0.73 (95\% CI 0.68, 0.77) for Black subjects. For dietary $\mathrm{Ca}$, the corresponding correlations were $0.53(95 \%$ CI $0.47,0.59)$ and $0.54(95 \%$ CI $0 \cdot 46,0 \cdot 60)$, respectively ${ }^{(25)}$.

\section{Cancer and death ascertainment}

Cancer cases were identified by computer-matching identifiers from Adventist Health Study-2 cohort members to those from State Cancer Registries. At the time of writing, matches had been made with all US states excepting Maine and Wisconsin. Most registries had complete data through 2011 at the time of matching, and a few through 2012. Supplementing this, a biennial mailed questionnaire contained questions about any new cancer diagnosis. If a self-reported cancer was not found in the registry linkage, further follow-up by telephone clarified whether it was a true cancer case or not. If a new cancer seemed possible, medical records were requested and reviewed by the principal investigator.

Deaths were ascertained by annual matching with the US National Death Index. As vital status for Canadian subjects is not yet available, Canadian subjects ( $n$ 3800) are not included in these analyses. New cancers of the colon and/or rectum were those diagnosed after baseline for each subject. Those with previous cancers were excluded. The cancer site was identified using the International Classification of Diseases-10 (ICD-10-CM). Cancers of the sigmoid colon were allocated to the rectum. Carcinomas in situ were not included as cases. 


\section{Statistical analysis}

All analyses were performed with the statistical software package SAS version 9.3 or the R computer language ${ }^{(26)}$. Multiple imputation was used to fill the small amounts (2-10\% for different variables) of missing dietary data, at the level of the FFQ question. This imputation was usually guided by a random sub-sample of originally missing data that were subsequently filled in by telephone contact ${ }^{(27)}$. Multivariate hazard rates and confidence intervals were calculated using five imputed data sets, and the five sets of $\beta$ coefficients and their estimated variances were used to form composite point estimates and variances ${ }^{(28)}$.

Multivariate Cox regression models were developed to estimate hazard ratios (HR) and 95\% CI. Attained age (ages during the period of observation) was the time variable and all models were left-censored so excluding person-time before the ages at entry. Person-time was counted through to a CRC outcome, the year end for cancer registry completion in the state of that subject's residence, or censoring for other reason (e.g. death, or rarely emigration, request to drop from the study).

A basic model including only the independent variables of interest, namely race and gender, was evaluated first. Other candidate covariates were selected based on review of the literature. These included: family history of CRC cancer (yes/no), education (high school or less, some college, at least college graduate), duration of vigorous activity (none, less than $60 \mathrm{~min} /$ week, at least $60 \mathrm{~min} /$ week), BMI (less than $25 \mathrm{~kg} / \mathrm{m}^{2}, 25$ to $30 \mathrm{~kg} / \mathrm{m}^{2}$, more than $30 \mathrm{~kg} / \mathrm{m}^{2}$ ), alcohol consumption within 2 years (yes/no), smoking status (ever/never), appropriate screening for CRC cancer (yes/no), personal history of peptic ulcer (yes/ no), personal history of polyps (yes/no), personal history of diabetes mellitus (yes/no), aspirin use, statin use (for both medications, at least weekly for at least the last 2 years; yes/no), fibre (grams of intake), unprocessed red meat, processed red meat, fish and poultry (grams of intake). Ca and total dairy were included in the same models as appropriate.

Nutrient composition was estimated using the Nutrition Data System for Research (NDS-R) 2008, a database updated annually while maintaining relevant information true to the version used for data collection ${ }^{(29)}$. Where commercially prepared food products were not found here, we created recipes to generate nutrient composition at the ingredient level.

For the CRC screening variable, participants over 50 years old and at low or average risk of developing CRC (based on absence of history of adenomatous polyps, inflammatory bowel disease, family history of CRC, polyps, a hereditary CRC syndrome), and who reported that they had been screened by a colonoscopy within the last 5 years, and/or faecal occult blood test within the last year, were considered compliant (American Cancer Society criteria). Those at higher risk of developing CRC were considered compliant if they reported screening before the age of 50 years old with colonoscopy every 1 to 2 years $^{(30)}$. A nested analysis among those who should have screened used an indicator variable to identify those at least 50 years of age or less than 50 but high risk, and then evaluated the product between this variable and screening status.

Dietary variables were log-transformed prior to model building. All non-zero dietary variables were energyadjusted (residual method), except for Ca from supplements, and modelled as continuous variables. Then, for each dairy nutrient, dairy foods and $\mathrm{Ca}$, the HR comparing mid-points of extreme quintiles ( $Q_{\text {upper }}$ and $\left.Q_{\text {lower }}\right)$ on risk of CRC was calculated by ( $\left.\mathrm{Qupper}_{\mathrm{Q}} / \mathrm{Q}_{\text {lower }}\right)^{\beta}$, where $\beta$ is the regression coefficient for the variable of interest. The energy adjustments were race specific as Black subjects consumed much less dairy on average and validity coefficients also varied somewhat by race.

Possible interactions between covariates and the relative risks of CRC at different levels of the exposures of interest were tested using product terms between the exposures of interest and these covariates. As no interaction terms were statistically significant, none were included in the final model. The dairy nutrients (kilocalories, protein, fat) were highly correlated with each other $(r \geq 0 \cdot 8)$. Therefore, individual models were built for each nutrient. All dairy foods, however, could be included in a single model since intercorrelations between them were relatively low.

Finally, using a concurrent second FFQ and repeated $24 \mathrm{~h}$ dietary recalls from our calibration study subjects ( $n$ 1011), who represent the cohort ${ }^{(25)}$, we formed new variables, E(Recall IFFQ data, covariates), for all dietary variables in selected models in a race-specific fashion. These data were then used for regression calibration ${ }^{(31,32)}$ of selected key analyses to at least partially correct for the effects of measurement errors. Confidence intervals for calibrated coefficients were estimated by the bootstrap BCa method ${ }^{(33)}$ ( $\left.n_{\text {samples }} 2000\right)$.

\section{Results}

During a mean follow-up of $7 \cdot 8$ years, 380 new cases of colon cancer and 111 cases of rectal cancer were identified.

Table 1 shows the baseline characteristics of the study population, comparing subjects at first, third and fifth quintiles of intake of dairy foods. Higher dairy intake was positively associated with BMI, alcohol use within 2 years, a smoking history, prevalent diabetes, use of aspirin and statins, consumption of each of the meats, and also Ca. Dairy intake was negatively associated with male sex, percentage of Black subjects, exercise, age, and fibre consumption.

Table 2 compares the same variables across the first, third and fifth quintiles of $\mathrm{Ca}$ consumption. Higher Ca consumption 
Table 1 Demographic characteristics by quintile of energy from total dairy intake in participants of the Adventist Health Study-2, USA, 2002-2011

\begin{tabular}{|c|c|c|c|c|c|c|c|}
\hline \multirow[b]{3}{*}{ Categorical variables } & \multicolumn{6}{|c|}{ Quintile of energy from total dairy intake } & \multirow[b]{3}{*}{$P$ value } \\
\hline & \multicolumn{2}{|c|}{ 1st $(n$ 15 481) } & \multicolumn{2}{|c|}{ 3rd (n 15 481) } & \multicolumn{2}{|c|}{ 5th $(n 15480)$} & \\
\hline & $n$ & $\%$ & $n$ & $\%$ & $n$ & $\%$ & \\
\hline Male & 5595 & $36 \cdot 1$ & 5598 & $36 \cdot 2$ & 4831 & $31 \cdot 2$ & $<0.001$ \\
\hline Race, Black & 4325 & $27 \cdot 9$ & 4811 & $31 \cdot 1$ & 2753 & $17 \cdot 8$ & $<0.001$ \\
\hline $\mathrm{BMI}\left(\mathrm{kg} / \mathrm{m}^{2}\right)$ & & & & & & & $<0.001$ \\
\hline$<25.0$ & 8766 & $56 \cdot 6$ & 5607 & $36 \cdot 2$ & 5176 & 33.4 & \\
\hline $25 \cdot 0-30 \cdot 0$ & 4535 & $29 \cdot 3$ & 5665 & $36 \cdot 6$ & 5520 & $35 \cdot 7$ & \\
\hline$>30.0$ & 2180 & $14 \cdot 1$ & 4209 & $27 \cdot 2$ & 4784 & 30.9 & \\
\hline Education & & & & & & & $<0.001$ \\
\hline High school or less & 3011 & $19 \cdot 4$ & 3310 & 21.4 & 3393 & 21.9 & \\
\hline Some college & 5954 & 38.5 & 6147 & $39 \cdot 7$ & 6312 & $40 \cdot 8$ & \\
\hline At least bachelor's degree & 6516 & $42 \cdot 1$ & 6024 & 38.9 & 5775 & $37 \cdot 3$ & \\
\hline Exercise (min/week) & & & & & & & $<0.001$ \\
\hline 0 & 2649 & $17 \cdot 1$ & 3225 & $20 \cdot 8$ & 3682 & $23 \cdot 8$ & \\
\hline$>0$ but $<60$ & 4697 & $30 \cdot 3$ & 5422 & $35 \cdot 0$ & 5428 & $35 \cdot 1$ & \\
\hline$\geq 60$ & 8135 & 52.5 & 6834 & $44 \cdot 1$ & 6370 & $41 \cdot 1$ & \\
\hline Family history of colon cancer & 1423 & $9 \cdot 2$ & 1428 & $9 \cdot 2$ & 1369 & $8 \cdot 8$ & 0.314 \\
\hline Polyps & 1056 & 6.8 & 1152 & 7.4 & 1203 & $7 \cdot 8$ & 0.019 \\
\hline ACS screening compliance & 5976 & 38.6 & 5892 & $38 \cdot 1$ & 5793 & 37.4 & 0.01 \\
\hline Alcohol use within 2 years of baseline & 559 & 3.6 & 1656 & $10 \cdot 7$ & 2498 & $16 \cdot 1$ & $<0.001$ \\
\hline Smoking (ever) & 2505 & $16 \cdot 2$ & 3245 & $21 \cdot 0$ & 3275 & $21 \cdot 2$ & $<0.001$ \\
\hline Diabetes & 677 & 4.4 & 1073 & 6.9 & 1050 & $6 \cdot 8$ & $<0.001$ \\
\hline Aspirin & 1665 & $10 \cdot 8$ & 2453 & $15 \cdot 8$ & 2788 & $18 \cdot 0$ & $<0.001$ \\
\hline Statin use in the last 5 years for $\geq 2$ years & 1177 & $7 \cdot 6$ & 1897 & $12 \cdot 3$ & 1934 & $12 \cdot 5$ & $<0.001$ \\
\hline Continuous variables & Mean & SD & Mean & SD & Mean & SD & $P$ value \\
\hline Age (years) & $58 \cdot 8$ & $14 \cdot 0$ & $56 \cdot 6$ & 14.1 & 56.5 & 14.5 & $<0.001$ \\
\hline Energy intake (kJ/d) & $7964 \cdot 7$ & $3336 \cdot 3$ & 8029.5 & 3292.4 & $7889 \cdot 3$ & $2734 \cdot 2$ & 0.002 \\
\hline Energy intake $(\mathrm{kcal} / \mathrm{d})$ & $1903 \cdot 6$ & 797.4 & $1919 \cdot 1$ & 786.9 & $1885 \cdot 6$ & 653.5 & 0.002 \\
\hline Fibre $(g / d)$ & 43.2 & $20 \cdot 2$ & 33.1 & $15 \cdot 6$ & $25 \cdot 7$ & 11.1 & $<0.001$ \\
\hline Processed red meat $(\mathrm{g} / \mathrm{d})$ & 0.1 & 1.5 & 0.8 & 3.0 & $1 \cdot 1$ & 3.6 & $<0.001$ \\
\hline Unprocessed red meat (g/d) & 1.2 & $9 \cdot 8$ & 7.6 & $17 \cdot 1$ & $12 \cdot 1$ & $20 \cdot 1$ & $<0.001$ \\
\hline Fish $(g / d)$ & $6 \cdot 1$ & $21 \cdot 0$ & $12 \cdot 8$ & 21.3 & $12 \cdot 0$ & $17 \cdot 8$ & $<0.001$ \\
\hline Poultry (g/d) & 3.3 & 14.9 & 11.9 & 20.4 & $14 \cdot 6$ & 20.7 & $<0.001$ \\
\hline Total Ca $(\mathrm{mg} / \mathrm{d})$ & 1157.5 & $635 \cdot 7$ & 1159.9 & 632.1 & $1486 \cdot 3$ & $726 \cdot 7$ & $<0.001$ \\
\hline Total dairy (kJ/d) & $123 \cdot 4$ & 91.6 & 635.1 & 325.9 & $1648 \cdot 9$ & $802 \cdot 5$ & $<0.001$ \\
\hline Total dairy (kcal/d) & 29.5 & 21.9 & 151.8 & 77.9 & 394.1 & 191.8 & $<0.001$ \\
\hline
\end{tabular}

ACS, American Cancer Society.

The $x^{2}$ test for homogeneity was used to compare quintiles of energy from total dairy intake for categorical variables, while ANOVA was used for comparisons of continuous variables.

was positively associated with education, exercise, family history of colon cancer, previous colon polyps, American Cancer Society screening compliance, use of aspirin and statins, age and dairy intake. Ca intake was negatively associated with male sex, percentage of Black subjects, BMI, a smoking history, and consumption of red meat and poultry.

Similar intakes of fibre, meat (including red meat), dairy nutrients (total, protein, fat, carbohydrate) and Ca (total, from diet, from diary and from supplements) were observed among cancer cases as compared with the cohort in univariate analyses (Table 3).

Proportional hazard regression analyses, associating intake of each selected dairy nutrient with risk of all CRC, cancers of the colon and rectum separately, are presented in Table 4. Results from regression calibration (measurement error-corrected) analyses gave support to a statistically significant inverse association between dairy intake (either adjusted for and hence independent of $\mathrm{Ca}$, or unadjusted) and the risk of rectal cancer $(\mathrm{HR}=0.31,95 \%$ CI 0.09, 0.88 when adjusted for
Ca), comparing extreme quintiles of intake as measured by the repeated recalls. In uncorrected analyses, comparing extreme quintiles of FFQ intake, rectal cancer again showed statistically significant inverse relationships with total energy from dairy intake whether this was adjusted for $\mathrm{Ca}$ or not $(\mathrm{HR}=0.57,95 \% \mathrm{CI} 0.33,0.97 ; P<0.041$ when adjusted for Ca). Similar but non-significant trends were seen with dairy fat, protein and less clearly carbohydrate.

Associations between risk of colon cancer and total dairy (corrected and uncorrected analyses), dairy protein, dairy fat and colon cancer in uncorrected analyses were mostly close to the null when comparing extreme quintiles of intake. When adjusting only for non-dairy $\mathrm{Ca}$ (hence the total dairy variable includes any $\mathrm{Ca}$ effect), the HR for colon cancer was 0.83 (but not statistically significant) in both corrected and uncorrected analyses. Significant protective associations between total dairy intake and risk of colorectal cancer, whether dairy was measured in $\mathrm{kcal} / \mathrm{d}(\mathrm{HR}=0.77,95 \%$ CI $0.59,0.99 ; P=0.048)$ or $\mathrm{g} / \mathrm{d}$ 
Table 2 Demographic characteristics by quintile of total calcium intake in participants of the Adventist Health Study-2, USA, 2002-2011

\begin{tabular}{|c|c|c|c|c|c|c|c|}
\hline \multirow[b]{3}{*}{ Categorical variables } & \multicolumn{6}{|c|}{ Quintile of total $\mathrm{Ca}$ intake } & \multirow[b]{3}{*}{$P$ value } \\
\hline & \multicolumn{2}{|c|}{ 1st $(n$ 15 481) } & \multicolumn{2}{|c|}{ 3rd (n 15 481) } & \multicolumn{2}{|c|}{ 5th $(n 15480)$} & \\
\hline & $n$ & $\%$ & $n$ & $\%$ & $n$ & $\%$ & \\
\hline Male & 7006 & $45 \cdot 3$ & 6185 & $40 \cdot 0$ & 2637 & $17 \cdot 0$ & $<0.001$ \\
\hline Race, Black & 5968 & $38 \cdot 6$ & 4431 & $28 \cdot 6$ & 2323 & $15 \cdot 0$ & $<0.001$ \\
\hline BMI $\left(\mathrm{kg} / \mathrm{m}^{2}\right)$ & & & & & & & $<0.001$ \\
\hline$<25.0$ & 5752 & $37 \cdot 2$ & 5934 & $38 \cdot 3$ & 7143 & $46 \cdot 1$ & \\
\hline $25 \cdot 0-30 \cdot 0$ & 5450 & $35 \cdot 2$ & 5551 & $35 \cdot 9$ & 5063 & $32 \cdot 7$ & \\
\hline$>30.0$ & 4279 & $27 \cdot 6$ & 3996 & $25 \cdot 8$ & 3274 & $21 \cdot 1$ & \\
\hline Education & & & & & & & $<0.001$ \\
\hline High school or less & 3833 & $24 \cdot 8$ & 3124 & $20 \cdot 2$ & 2909 & $18 \cdot 8$ & \\
\hline Some college & 6149 & 39.7 & 5948 & 38.4 & 6474 & 41.8 & \\
\hline At least bachelor's degree & 5499 & 35.5 & 6409 & 41.4 & 6097 & 39.4 & \\
\hline Exercise ( $\mathrm{min} /$ week) & & & & & & & $<0.001$ \\
\hline 0 & 3863 & $25 \cdot 0$ & 2902 & 18.7 & 3137 & $20 \cdot 3$ & \\
\hline$>0$ but $<60$ & 5758 & $37 \cdot 2$ & 5319 & $34 \cdot 4$ & 4576 & $29 \cdot 6$ & \\
\hline$\geq 60$ & 5860 & 37.9 & 7260 & $46 \cdot 9$ & 7767 & $50 \cdot 2$ & \\
\hline Family history of colon cancer & 1305 & 8.4 & 1338 & 8.6 & 1592 & $10 \cdot 3$ & $<0.001$ \\
\hline Polyps & 928 & $6 \cdot 0$ & 1093 & $7 \cdot 1$ & 1412 & $9 \cdot 1$ & $<0.001$ \\
\hline ACS screening compliance & 4745 & 30.7 & 5537 & $35 \cdot 8$ & 7603 & $49 \cdot 1$ & $<0.001$ \\
\hline Alcohol use within 2 years of baseline & 1666 & $10 \cdot 8$ & 1530 & 9.9 & 1726 & $11 \cdot 1$ & $<0.001$ \\
\hline Smoking (ever) & 3736 & $24 \cdot 1$ & 2958 & $19 \cdot 1$ & 2655 & $17 \cdot 2$ & $<0.001$ \\
\hline Diabetes & 902 & $5 \cdot 8$ & 1045 & $6 \cdot 8$ & 858 & 5.5 & $<0.001$ \\
\hline Aspirin & 1796 & $11 \cdot 6$ & 2311 & 14.9 & 2972 & $19 \cdot 2$ & $<0.001$ \\
\hline Statin use in the last 5 years for $\geq 2$ years & 1533 & $9 \cdot 9$ & 1766 & 11.4 & 1972 & $12 \cdot 7$ & $<0.001$ \\
\hline Continuous variables & Mean & SD & Mean & SD & Mean & SD & $P$ value \\
\hline Age (years) & 54.9 & $13 \cdot 8$ & $56 \cdot 2$ & 14.5 & 60.5 & 13.4 & $<0.001$ \\
\hline Energy intake (kJ/d) & 7294.4 & $3664 \cdot 3$ & $8614 \cdot 0$ & $3140 \cdot 5$ & $7200 \cdot 2$ & $2475 \cdot 7$ & $<0.001$ \\
\hline Energy intake $(\mathrm{kcal} / \mathrm{d})$ & $1743 \cdot 4$ & $875 \cdot 8$ & $2058 \cdot 8$ & $750 \cdot 6$ & $1720 \cdot 9$ & $591 \cdot 7$ & $<0.001$ \\
\hline Fibre $(g / d)$ & $29 \cdot 1$ & $18 \cdot 1$ & 36.9 & $17 \cdot 3$ & 31.2 & 13.7 & $<0.001$ \\
\hline Processed red meat $(\mathrm{g} / \mathrm{d})$ & $1 \cdot 1$ & 4.0 & 0.6 & $2 \cdot 9$ & 0.4 & 1.9 & $<0.001$ \\
\hline Unprocessed red meat $(\mathrm{g} / \mathrm{d})$ & 9.4 & $21 \cdot 1$ & $6 \cdot 8$ & $16 \cdot 3$ & $5 \cdot 3$ & $12 \cdot 8$ & $<0.001$ \\
\hline Fish $(g / d)$ & $10 \cdot 9$ & 20.5 & $12 \cdot 0$ & $23 \cdot 8$ & $9 \cdot 2$ & $16 \cdot 3$ & $<0.001$ \\
\hline Poultry $(\mathrm{g} / \mathrm{d})$ & $12 \cdot 6$ & $24 \cdot 1$ & $10 \cdot 6$ & $19 \cdot 8$ & 8.7 & $16 \cdot 2$ & $<0.001$ \\
\hline Total Ca (mg/d) & 577.3 & $284 \cdot 1$ & $1147 \cdot 6$ & $397 \cdot 0$ & $2057 \cdot 6$ & 578.0 & $<0.001$ \\
\hline Total dairy (kJ/d) & $480 \cdot 3$ & 461.1 & 823.0 & $668 \cdot 2$ & 871.9 & $845 \cdot 2$ & $<0.001$ \\
\hline Total dairy (kcal/d) & 114.8 & $110 \cdot 2$ & $196 \cdot 7$ & 159.7 & $208 \cdot 4$ & $202 \cdot 0$ & $<0.001$ \\
\hline
\end{tabular}

ACS, American Cancer Society.

The $x^{2}$ test for homogeneity was used to compare quintiles of Ca intake for categorical variables, while ANOVA was used for comparisons of continuous variables.

(HR $=0.75,95 \%$ CI $0.57,0.98 ; P=0.037)$, were seen when adjusted for only non-dairy $\mathrm{Ca}$, thus leaving a total dairy variable that includes any effects of dairy Ca. Significant protective associations between dairy variables and rectal cancer were not clearly seen when Black subjects were analysed separately (data not shown). However, with only twenty-five rectal cancer cases, no conclusions can be drawn. Supplemental Table 1 (see online supplementary material) shows corresponding results for each quintile of intake.

When analysing the nominated extreme intakes (usually quintiles) of specific dairy foods, evaluated independently and adjusting for supplemental and non-dairy Ca (Table 5), no significant effects were seen for cheese, cottage cheese or yoghurt. However, for milk, the results for risks of CRC (HR $=0.63,95 \%$ CI $0.43,0.89 ; P=0.013)$ and colon cancer ( $\mathrm{HR}=0.67,95 \%$ CI $0.45,0.99 ; P=0.047)$ were statistically significant, and that for rectal cancer trended in the same direction $(\mathrm{HR}=0.52,95 \% \mathrm{CI} 0 \cdot 24,1 \cdot 10 ; P=0 \cdot 091)$. Supplemental Table 2 (see online supplementary material) shows corresponding results for each quintile of intake.
Table 6 presents results of proportional hazard regression models where the exposures are total $\mathrm{Ca}$ intake, also particular sources of $\mathrm{Ca}$, comparing the nominated extreme intakes (usually quintiles). Measurement error-corrected analyses gave significant support for the existence of a significant inverse association between total $\mathrm{Ca}$ intake and risk of colon cancer, whether adjusted for dairy energy or not $(\mathrm{HR}=0.55$, $95 \%$ CI 0.28, 0.98 when adjusted for dairy energy), comparing extreme quintiles of repeated dietary recall data. In uncorrected analyses, a statistically significant association was observed when comparing extreme quintiles of intake of total $\mathrm{Ca}$, for risk of colorectal and colon cancers, and associations with total, dietary and non-dairy $\mathrm{Ca}$ were borderline significant when dairy energy was adjusted for. Associations between total and dietary sources of $\mathrm{Ca}$ and risk of rectal cancer were non-significant, either closer to the null or trended in the positive direction, with the exception of dairy $\mathrm{Ca}$ that was not adjusted for dairy energy (due to severe collinearity) and hence included non-Ca components of dairy. Supplemental Ca was significantly negatively associated with 
Table 3 Mean intakes of dairy and calcium by cancer of the colon and rectum among participants of the Adventist Health Study-2, USA, 2002-2011

\begin{tabular}{|c|c|c|c|c|c|c|c|}
\hline \multirow[b]{2}{*}{ Dietary variable } & \multirow[b]{2}{*}{ Whole cohort } & \multicolumn{2}{|c|}{ Colorectal cancer } & \multicolumn{2}{|c|}{ Colon cancer } & \multicolumn{2}{|c|}{ Rectal cancer } \\
\hline & & Cases & $P$ value* & Cases & $P$ value* & Cases & $P$ value ${ }^{*}$ \\
\hline \multicolumn{8}{|l|}{ Mean intake of dairy } \\
\hline Total dairy (kJ/d) & 743.9 & 728.4 & 0.6 & $750 \cdot 6$ & 0.9 & 651.0 & 0.2 \\
\hline Total dairy $(\mathrm{kccl} / \mathrm{d})$ & 177.8 & $174 \cdot 1$ & 0.6 & $179 \cdot 4$ & 0.9 & 155.6 & 0.2 \\
\hline Total dairy $(\mathrm{g} / \mathrm{d})$ & 188.7 & $190 \cdot 6$ & 0.9 & $198 \cdot 2$ & 0.4 & 164.5 & 0.3 \\
\hline Protein $(\mathrm{g} / \mathrm{d})$ & 9.5 & 9.5 & 0.99 & 9.9 & 0.5 & 8.3 & 0.2 \\
\hline Fat $(\mathrm{g} / \mathrm{d})$ & $10 \cdot 8$ & $10 \cdot 4$ & 0.4 & $10 \cdot 7$ & 0.8 & $9 \cdot 6$ & 0.2 \\
\hline Carbohydrate $(\mathrm{g} / \mathrm{d})$ & 11.0 & $10 \cdot 9$ & 0.8 & $11 \cdot 3$ & 0.7 & $9 \cdot 3$ & 0.2 \\
\hline \multicolumn{8}{|l|}{ Mean intake of $\mathrm{Ca}$} \\
\hline Total Ca (mg/d) & 1235.0 & 1231.8 & 0.9 & 1248.4 & 0.7 & $1175 \cdot 0$ & 0.3 \\
\hline Diet $\mathrm{Ca}(\mathrm{mg} / \mathrm{d})$ & $885 \cdot 3$ & $869 \cdot 8$ & 0.4 & 874.0 & 0.6 & $855 \cdot 1$ & 0.5 \\
\hline Dairy Ca $(\mathrm{mg} / \mathrm{d})$ & 283.4 & $276 \cdot 0$ & 0.6 & $285 \cdot 8$ & 0.9 & 242.4 & 0.2 \\
\hline Supplemental Ca $(\mathrm{mg} / \mathrm{d})$ & 349.7 & $362 \cdot 0$ & 0.6 & 374.4 & 0.3 & $319 \cdot 8$ & 0.5 \\
\hline
\end{tabular}

*Testing null hypothesis of no difference between cases and the whole cohort.

Table 4 Multivariate-adjusted (adj.) uncorrected and regression calibration (RC) hazard ratios (HR) describing associations between dairy intake and incident cancers of the colon and rectum among participants of the Adventist Health Study-2, USA, 2002-2011

\begin{tabular}{|c|c|c|c|c|c|c|c|}
\hline \multirow[b]{2}{*}{ Dairy nutrients } & \multirow[b]{2}{*}{ Medians of extreme quintiles ${ }^{*}$} & \multicolumn{2}{|c|}{ Colorectal cancer } & \multicolumn{2}{|c|}{ Colon cancer } & \multicolumn{2}{|c|}{ Rectal cancer } \\
\hline & & $\mathrm{HR}$ & $95 \% \mathrm{Cl}$ & HR & $95 \% \mathrm{Cl}$ & $\mathrm{HR}$ & $95 \% \mathrm{Cl}$ \\
\hline \multirow[t]{2}{*}{ Total dairy (kcal/d) (not adj. for Ca)† } & $114.2 \mathrm{~kJ} / \mathrm{d}(27.30 \mathrm{kcal} / \mathrm{d})$ & & 1.00 & & 1.00 & & 1.00 \\
\hline & $1422.6 \mathrm{~kJ} / \mathrm{d}(340.00 \mathrm{kcal} / \mathrm{d})$ & 0.78 & $0.60,1.00$ & 0.87 & $0.64,1 \cdot 17$ & 0.56 & $0.33,0.94$ \\
\hline \multirow[t]{2}{*}{ Total dairy $(\mathrm{kcal} / \mathrm{d})($ adj. for total $\mathrm{Ca}) \ddagger$} & $114.2 \mathrm{~kJ} / \mathrm{d}(27.30 \mathrm{kcal} / \mathrm{d})$ & & 1.00 & & 1.00 & & 1.00 \\
\hline & $1422.6 \mathrm{~kJ} / \mathrm{d}(340.00 \mathrm{kcal} / \mathrm{d})$ & 0.83 & $0.63,1.09$ & 0.94 & $0.69,1.30$ & 0.57 & $0.33,0.97$ \\
\hline \multirow[t]{2}{*}{ Total dairy (kcal/d) (adj. for non-dairy Ca)‡ } & $114.2 \mathrm{~kJ} / \mathrm{d}(27.30 \mathrm{kcal} / \mathrm{d})$ & & 1.00 & & 1.00 & & 1.00 \\
\hline & $1422.6 \mathrm{~kJ} / \mathrm{d}(340.00 \mathrm{kcal} / \mathrm{d})$ & 0.77 & $0.59,0.99$ & 0.83 & $0.61,1.13$ & 0.60 & $0.35,1.02$ \\
\hline \multirow[t]{2}{*}{ Total dairy $(\mathrm{kcal} / \mathrm{d})$ (not adj. for $\mathrm{Ca})-\mathrm{RC} \dagger, \S$} & $75.7 \mathrm{~kJ} / \mathrm{d}(18.10 \mathrm{kcal} / \mathrm{d})$ & & 1.00 & & 1.00 & & 1.00 \\
\hline & $1077.0 \mathrm{~kJ} / \mathrm{d}(257.40 \mathrm{kcal} / \mathrm{d})$ & 0.67 & $0.37,1.11$ & 0.86 & $0.46,1.62$ & 0.31 & $0.09,0.96$ \\
\hline \multirow[t]{2}{*}{ Total dairy (kcal/d) (adj. for total Ca) - RC $\ddagger$,§ } & $75.7 \mathrm{~kJ} / \mathrm{d}(18.10 \mathrm{kcal} / \mathrm{d})$ & & 1.00 & & 1.00 & & 1.00 \\
\hline & $1077.0 \mathrm{~kJ} / \mathrm{d}(257.40 \mathrm{kcal} / \mathrm{d})$ & $0 \cdot 77$ & $0.41,1.31$ & 1.03 & $0.50,1.94$ & 0.31 & $0.09,0.88$ \\
\hline \multirow[t]{2}{*}{ Total dairy (kcal/d) (adj. for non-dairy Ca) - RC‡,§ } & $75.7 \mathrm{~kJ} / \mathrm{d}(18.10 \mathrm{kcal} / \mathrm{d})$ & & 1.00 & & 1.00 & & 1.00 \\
\hline & $1077.0 \mathrm{~kJ} / \mathrm{d}(257.40 \mathrm{kcal} / \mathrm{d})$ & 0.69 & $0.36,1 \cdot 17$ & 0.83 & $0.39,1.49$ & 0.40 & $0.11,1.27$ \\
\hline \multirow[t]{2}{*}{ Total dairy (g/d) (adj. for non-dairy Ca)‡ } & $9.90 \mathrm{~g} / \mathrm{d}$ & & 1.00 & & 1.00 & & 1.00 \\
\hline & $415.00 \mathrm{~g} / \mathrm{d}$ & 0.75 & $0.57,0.98$ & 0.80 & $0.58,1.09$ & 0.61 & $0.35,1.07$ \\
\hline \multirow[t]{2}{*}{ Dairy protein $\|$} & $1.04 \mathrm{~g} / \mathrm{d}$ & & 1.00 & & 1.00 & & 1.00 \\
\hline & $19 \cdot 70 \mathrm{~g} / \mathrm{d}$ & 0.80 & $0.60,1.06$ & 0.86 & $0.62,1.18$ & 0.62 & $0.33,1.14$ \\
\hline \multirow[t]{2}{*}{ Dairy fat $\|$} & $1.90 \mathrm{~g} / \mathrm{d}$ & & 1.00 & & 1.00 & & 1.00 \\
\hline & $20.50 \mathrm{~g} / \mathrm{d}$ & 0.81 & $0.61,1.09$ & 0.89 & $0.64,1.25$ & 0.60 & $0.32,1 \cdot 13$ \\
\hline \multirow[t]{2}{*}{ Dairy carbohydrate\| } & $1.10 \mathrm{~g} / \mathrm{d}$ & & 1.00 & & 1.00 & & 1.00 \\
\hline & $24.4 \mathrm{~g} / \mathrm{d}$ & 0.78 & $0.59,1.02$ & 0.80 & $0.59,1.09$ & 0.70 & $0.39,1.27$ \\
\hline
\end{tabular}

*Except for RC analyses, quintiles are from FFQ data.

†Adjusted for fibre, unprocessed red meat, processed red meat, fish, poultry, gender, race, BMI, education, alcohol consumption, cigarette smoking history, diabetes, use of aspirin, use of statin, physical activity, family history of colorectal cancer, history of polyps, screening for colorectal cancer.

$\ddagger$ Adjusted in addition for either total $\mathrm{Ca}$ or only supplemental and non-dairy $\mathrm{Ca}$ (as indicated).

$\S R C$ analyses. Units are $\mathrm{kcal} / \mathrm{d}$ and quintiles are those from repeated recalls, as is appropriate for the RC.

|| Dairy nutrient variables are assessed individually in the regression models.

risk of $\mathrm{CRC}(\mathrm{HR}=0 \cdot 80,95 \% \mathrm{CI} 0 \cdot 65,0 \cdot 98 ; P=0 \cdot 034)$; this also being nearly significant for both rectal and colon cancer end points while adjusting for dairy energy and dietary Ca. Supplemental Table 3 (see online supplementary material) shows corresponding results for each quintile of intake.

\section{Discussion}

To summarize these findings, it is clear that dairy and/or Ca intake and milk consumption are significantly negatively associated with risk of CRC in these data. Risk of colon cancer appears to be most strongly associated with $\mathrm{Ca}$ intake and not with dairy intake when adjusted for total $\mathrm{Ca}$ intake. Findings for rectal cancer have wider confidence intervals and are therefore less secure, but this cancer appears to be negatively associated with dairy intake, and more clearly with the non-Ca dairy components. The rectal cancer association with dairy is most significant after adjusting for total $\mathrm{Ca}$, and neither total $\mathrm{Ca}$ nor non-dairy $\mathrm{Ca}$ showed a protective association after adjustment for dairy intake. Milk intake was significantly associated with risk of colon cancer and also strongly associated with rectal cancer (but here only borderline 
Table 5 Multivariate ${ }^{*}$-adjusted hazard ratios (HR) of the associations between dairy foods and incident cancers of the colon and rectum among participants of the Adventist Health Study-2, USA, 2002-2011

\begin{tabular}{|c|c|c|c|c|c|c|c|}
\hline \multirow[b]{2}{*}{ Dairy foods $†$} & \multirow{2}{*}{$\begin{array}{l}\text { Medians of extreme } \\
\text { quintiles }(\mathrm{g} / \mathrm{d})\end{array}$} & \multicolumn{2}{|c|}{ Colorectal cancer } & \multicolumn{2}{|c|}{ Colon cancer } & \multicolumn{2}{|c|}{ Rectal cancer } \\
\hline & & $\mathrm{HR}$ & $95 \% \mathrm{Cl}$ & $\mathrm{HR}$ & $95 \% \mathrm{Cl}$ & $\mathrm{HR}$ & $95 \% \mathrm{Cl}$ \\
\hline \multirow[t]{2}{*}{ Cheese } & 0.10 & \multicolumn{2}{|c|}{1.00} & \multicolumn{2}{|c|}{1.00} & \multicolumn{2}{|r|}{1.00} \\
\hline & 24.80 & 1.00 & $0.69,1.46$ & 1.03 & $0.68,1.58$ & 0.91 & $0.40,2.07$ \\
\hline \multirow[t]{2}{*}{ Cottage cheese } & $0.00 \S$ & \multicolumn{2}{|c|}{1.00} & \multicolumn{2}{|c|}{1.00} & \multicolumn{2}{|r|}{1.00} \\
\hline & $46 \cdot 50$ & 1.05 & $0.78,1.40$ & 1.04 & $0.74,1.45$ & 1.06 & $0.57,1.93$ \\
\hline \multirow[t]{2}{*}{ Milk } & $5 \cdot 20$ & \multicolumn{2}{|c|}{1.00} & \multicolumn{2}{|c|}{1.00} & \multicolumn{2}{|r|}{1.00} \\
\hline & $\begin{array}{c}378.00 \\
0.00 \S\end{array}$ & 0.63 & $0.43,0.89$ & 1.00 & $\begin{array}{l}0.45,0.99 \\
00\end{array}$ & & $1.00^{0.24,1.10}$ \\
\hline Yoghurt & 105.00 & $1 \cdot 34$ & $1.00,1.81$ & 1.31 & $0.93,1.84$ & 1.49 & $0.80,2.77$ \\
\hline
\end{tabular}

${ }^{*}$ Adjusted for supplemental $\mathrm{Ca}$, non-dairy $\mathrm{Ca}$, fibre, unprocessed red meat, processed red meat, fish, poultry, gender, race, BMI, education, alcohol consumption, cigarette smoking history, diabetes, use of aspirin, use of statin, physical activity, family history of colorectal cancer, history of polyps, screening for colorectal cancer.

†Exposures adjusted for each other.

†Excluding cottage cheese.

§Compares zero intake with the median of the upper quartile of non-zero intakes.

Table 6 Multivariate-adjusted (adj.) uncorrected and regression calibration (RC) hazard ratios (HR) describing associations between calcium intake and incident cancers of the colon and rectum among participants of the Adventist Health Study-2, USA, 2002-2011

\begin{tabular}{|c|c|c|c|c|c|c|c|}
\hline \multirow[b]{2}{*}{ Calcium } & \multirow{2}{*}{$\begin{array}{l}\text { Medians of extreme } \\
\text { quintiles* }(\mathrm{mg} / \mathrm{d})\end{array}$} & \multicolumn{2}{|c|}{ Colorectal cancer } & \multicolumn{2}{|c|}{ Colon cancer } & \multicolumn{2}{|c|}{ Rectal cancer } \\
\hline & & $\mathrm{HR}$ & $95 \% \mathrm{Cl}$ & $\mathrm{HR}$ & $95 \% \mathrm{Cl}$ & $\mathrm{HR}$ & $95 \% \mathrm{Cl}$ \\
\hline \multirow{2}{*}{ Total $\mathrm{Ca}$ (not adj. for dairy energy)† } & 587.00 & & 1.00 & & 1.00 & & 1.00 \\
\hline & 1999.00 & 0.75 & $0.59,0.97$ & 0.74 & $0.56,0.98$ & 0.79 & $0.47,1.35$ \\
\hline \multirow{2}{*}{ Total Ca (adj. for dairy energy)‡ } & 587.00 & & 1.00 & & 1.00 & & 1.00 \\
\hline & 1999.00 & 0.79 & $0.61,1.03$ & 0.75 & $0.56,1.01$ & 0.93 & $0.54,1.58$ \\
\hline \multirow[t]{2}{*}{ Total Ca (not adj. for dairy energy - $\mathrm{RC}+, \S$} & $462 \cdot 00$ & & 1.00 & & 1.00 & & 1.00 \\
\hline & 1759.00 & 0.59 & $0.32,0.99$ & 0.55 & $0.28,0.98$ & 0.74 & $0.21,2.19$ \\
\hline \multirow[t]{2}{*}{ Total $\mathrm{Ca}$ (adj. for dairy energy) - $\mathrm{RC} \ddagger, \S$} & $462 \cdot 00$ & & 1.00 & & 1.00 & & 1.00 \\
\hline & $1759 \cdot 00$ & 0.63 & $0.36,1.06$ & 0.55 & $0.28,0.98$ & 1.04 & $0.33,4.42$ \\
\hline \multirow[t]{2}{*}{ Diet $\mathrm{Ca} \|$} & 530.00 & & 1.00 & & 1.00 & & 1.00 \\
\hline & $1196 \cdot 00$ & 0.89 & $0.69,1 \cdot 16$ & 0.78 & $0.58,1.06$ & 1.32 & $0.80,2.19$ \\
\hline \multirow[t]{2}{*}{ Dairy Caף } & 31.60 & & 1.00 & & 1.00 & & 1.00 \\
\hline & $590 \cdot 00$ & 0.77 & $0.60,1.00$ & 0.83 & $0.62,1.11$ & 0.62 & $0.36,1.05$ \\
\hline \multirow[t]{2}{*}{ Supplemental Ca\| } & 0.00 & & 1.00 & & 1.00 & & 1.00 \\
\hline & 1193.00 & 0.80 & $0.65,0.98$ & 0.83 & $0.65,1.05$ & 0.69 & $0.44,1.08$ \\
\hline \multirow[t]{2}{*}{ Non-dairy Caף } & 328.00 & & 1.00 & & 1.00 & & 1.00 \\
\hline & 859.00 & 0.87 & $0.64,1.17$ & 0.73 & $0.52,1.04$ & 1.44 & $0.81,2.58$ \\
\hline
\end{tabular}

${ }^{\star}$ Except for RC analyses, quintiles are from FFQ data. However, for supplemental Ca compares zero intake with the median of the upper quartile of non-zero supplemental $\mathrm{Ca}$.

†Model 1: total $\mathrm{Ca}$, fibre, unprocessed red meat, processed red meat, fish, poultry, gender, race, BMI, education, alcohol consumption, cigarette smoking history, diabetes, use of aspirin, use of statin, physical activity, family history of colorectal cancer, history of polyps, screening for colorectal cancer.

¥Model 2: Model 1 with addition of dairy energy.

$\S$ Quintiles are from the repeated dietary recall data as is appropriate for the RC.

IIModel 3: Ca from diet and $\mathrm{Ca}$ from supplements replace total $\mathrm{Ca}$ as separate variables. Other covariates are identical to Model 2.

TModel 4: Ca from dairy, non-dairy $\mathrm{Ca}$ and $\mathrm{Ca}$ from supplements are the three calcium variables, and dairy energy is omitted. Other covariates are identical to Model 2.

significant). This presumably reflects its $\mathrm{Ca}$ and non-Ca components. Supplemental Ca was also significantly associated with risk of $\mathrm{CRC}$, there not being a clear difference between colon and rectal cancers in this case. However, this somewhat surprising result for rectal cancer may be due to chance $(P=0 \cdot 105)$. Regression calibration applied to the major results revealed stronger associations as expected and generally preserved statistical significance. In our data, the magnitudes of the significant regression calibration HR estimates were substantial, in the range of 0.31 to 0.55 , when comparing extreme quintiles.
The association between dairy intake and the risk of CRC has been explored in other epidemiological studies ${ }^{(16-19,34-36)}$. The evidence generally favours a protective effect of dairy. However, most studies appear to have used models including either $\mathrm{Ca}$ or dairy alone, rather than evaluating both independently. Hence from those results, a dairy effect, a Ca effect, or both, is possible. Of the dairy foods, associations with milk have been most consistent as we found, although some studies disagree.

Higher consumption of total dairy products has been associated with reduced risk of CRC (both colon and rectal cancers) in the EPIC (European Prospective Investigation 
into Cancer and Nutrition) cohort study ${ }^{(16)}$ and a cohort of Swedish men ${ }^{(35)}$. A large Finnish cohort study also indicated that milk protein and the consumption of milk products were inversely associated with CRC risk ${ }^{(37)}$. In another Swedish cohort, greater intake of high-fat dairy foods was associated with a reduced risk of CRC, a protective effect that was stronger for distal colon cancer, but was not seen for rectal cancer. Considering individual dairy foods, the strongest inverse association was observed for high consumption of cheese ${ }^{(38)}$. Findings from other studies on the relationship between cheese consumption and $\mathrm{CRC}$ risk have mostly been null ${ }^{(39)}$. Furthermore, a systematic review and meta-analysis of nineteen cohort studies ${ }^{(36)}$ found that high intakes of total dairy products were associated with a statistically significant reduction in CRC risk and there was evidence for an inverse association with milk but not cheese or other dairy products. When stratified to colon and rectal sites the inverse associations were only present for colon cancer. These inconsistent results may be due to variable confounding between dairy and $\mathrm{Ca}$ (which cannot be determined from the published data) or perhaps to chance.

Biologically, a protective effect of dairy may be explained by its relatively high content of Ca, vitamin D, lactoferrin and conjugated linoleic acid (CLA). Each of these has been postulated to protect against the development of $\mathrm{CRC}^{(40)}$. Lactoferrin is an Fe-binding antimicrobial protein present in exocrine secretions such as milk ${ }^{(40)}$. As found in animal studies, this glycoprotein may enhance CRC mucosal immunity and decrease inflammation-related malignant transformation ${ }^{(41)}$. Lactoferrin also increases apoptosis and the expression of Fas in the colon mucosa during both early and late stages of carcinogenesis in rats. Fas is a member of the TNF receptor family $^{(42)}$. It thus may act as a chemo-preventive factor for carcinogenesis. Lipid components of dairy products, including butyric acid and CLA, may protect against tumorigenesis $^{(40)}$. Butyric acid is a major metabolite in colonic lumen that possesses anti-inflammatory and antitumour properties ${ }^{(43)}$. Milk lipid is the main dietary source of CLA. Studies of cultured cell lines have shown that CLA may inhibit the proliferation of CRC tumour cells by modifying the fluidity of cell membranes, reducing the synthesis of prostaglandins and stimulating the immune response ${ }^{(40)}$. Amounts of these substances may differ greatly in different dairy foods depending on fat content (butyric acid, CLA) $^{(44)}$, protein content (lactoferrin) ${ }^{(45)}$ and bacterial activity. Differences in the colonic microbiome between vegans and lacto-ovo vegetarians ${ }^{(46)}$ may also result from differences in dairy consumption.

Several epidemiological studies have found a 14 to $45 \%$ lower risk of $\mathrm{CRC}$ in subjects with at least moderate intakes of Ca $(\geq 700 \mathrm{mg} / \mathrm{d})^{(34,37,47,48)}$. However, in one US study this benefit appeared to plateau, or level off, at an intake of approximately $1200 \mathrm{mg} / \mathrm{d}^{(34)}$. It has been reported that a protective association with Ca intake seemed more evident for distal than proximal colon cancer ${ }^{(47,48)}$. Inconsistencies between findings are particularly evident when assessing sources of this mineral. One study found that total Ca intake of greater than $1445 \mathrm{mg} / \mathrm{d}$, compared with less than $956 \mathrm{mg} /$ d, was associated with a statistically significant $32 \%$ decrease in risk of CRC and a 39\% decrease in rectal cancer, and that dietary Ca was also associated with decreased risk of $\mathrm{CRC}^{(35)}$. Ca from supplements has also been associated with reduced risk of CRC among people who consumed $\geq 500 \mathrm{mg} / \mathrm{d}^{(34)}$. In the Iowa Women's Health Study of postmenopausal women, Ca intake of $\geq 1280 \mathrm{mg} / \mathrm{d}$ from both diet and supplements combined was associated with a $41 \%$ reduction in risk of rectal cancer ${ }^{(49)}$.

Ca has been shown to reduce proliferation, stimulate cellular differentiation, and induce apoptosis in cells of the gastrointestinal tract. Additionally, the binding of Ca to bile and fatty acids in the gastrointestinal tract has been hypothesized to reduce damage to the large bowel mucosa ${ }^{(50)}$.

Is it possible that these associations, as is suggested by our results, could be rather different in the rectum as compared with the colon? The rectal mucosa is exposed to the final faecal products (including bacterial products) for longer periods than the colonic mucosa as the rectum is a storage organ. Conceivably, colonic bacterial products resulting from interactions with residual dairy components may not be fully expressed until the rectum.

Strengths of the present study are its prospective design, the validation of new colon and rectum cancers by matching to cancer registries or by review of medical records, and the unique lifestyle of the Adventist Health Study-2 population with its wide variety of dietary habits. Many vegetarians obtain much Ca from non-dairy sources. Thus, collinearity between dairy and $\mathrm{Ca}$ is reduced. Few other Western cohorts have large numbers consuming no dairy or very low dairy. This produces extreme quintiles of dairy that are far apart, which also increases statistical power and reduces the likelihood of confounding by non-dietary factors. Another strength is the representative calibration study that allows application of regression calibration as a measurement error correction strategy. Finally, the Adventist Health Study-2 cohort is a sociodemographically diverse population, aside from membership in the Seventh-day Adventist Church, potentially producing results that have general application.

Limitations of our study include unavoidable inaccuracies in the assessment of food consumption, although published results comparing questionnaire with six $24 \mathrm{~h}$ dietary recalls show relatively good validity correlation for both the dairy foods ${ }^{(23)}$ and nutrients ${ }^{(25)}$ that are the focus of the current paper. Any such misclassification should be non-differential, usually biasing the results towards the null. The regression calibration analyses partially allay this concern. These results come from a population where about half are vegetarian, which must be considered when generalizing results. However, we did adjust for obvious differences from the general population in the analyses. 


\section{Conclusion}

Dairy intake, independent of Ca intake, was associated with a decreased risk of rectal cancer, and Ca intake was associated with decreased risk of colon cancer independent of dairy intake. Milk intake and supplemental Ca also gave evidence of protection although not clearly differing between the two cancer sites. Inconsistencies in other work may result from confounding between $\mathrm{Ca}$ and dairy. Further studies are needed to confirm these independent associations of $\mathrm{Ca}$ and dairy products, and to further assess possible differences between associated risks of colon and rectal cancers.

\section{Acknowledgements}

Acknowledgements: The authors thank the following cancer registries for their collaboration: 'Alaska Cancer Registry', 'Alabama Statewide Cancer Registry', 'Arizona Cancer Registry', 'Arkansas Central Cancer Registry', 'California Cancer Registry', 'Colorado Central Cancer Registry', 'Connecticut Tumor Registry', 'District of Columbia Cancer Registry', 'Delaware Cancer Registry', 'Florida Cancer Data System', 'Georgia Comprehensive Cancer Registry', 'Hawaii Tumor Registry', 'Cancer Data Registry of Idaho', 'Iowa Cancer Registry', 'Illinois State Cancer Registry', 'Indiana State Cancer Registry', 'Kansas Cancer Registry', 'Kentucky Cancer Registry', 'Louisiana Tumor Registry', 'Maryland Cancer Registry', 'Massachusetts Cancer Registry', 'Michigan Cancer Surveillance System', 'Minnesota Cancer Surveillance System', 'Mississippi Cancer Registry', 'Missouri Cancer Registry and Research Center', 'Montana Central Tumor Registry', 'Nebraska Cancer Registry', 'Nevada Central Cancer Registry', 'New Hampshire State Cancer Registry', 'New Jersey State Cancer Registry', 'New Mexico Tumor Registry', 'New York State Cancer Registry', 'North Carolina Central Cancer Registry', 'North Dakota Statewide Cancer Registry', 'Ohio Cancer Incidence Surveillance System', 'Oklahoma Central Cancer Registry', 'Oregon State Cancer Registry', 'Pennsylvania Cancer Registry', 'Rhode Island Cancer Registry', 'South Carolina Central Cancer Registry', 'South Dakota Cancer Registry', 'Tennessee Cancer Registry', 'Texas Cancer Registry', 'Utah Cancer Registry, NCI Contract HHSN261201300071', 'Vermont Cancer Registry', 'Virginia Cancer Registry', 'Washington State Cancer Registry', 'West Virginia Cancer Registry' and 'Wyoming Cancer Surveillance Program'. Financial support: This study was supported by the National Institutes of Health (grant number U01CA152939); the National Institute of Food and Agriculture (grant number 2010-38938-20924); and the World Cancer Research Fund (UK) (grant number 2009/93). The funders had no role in the design, analysis or writing of this article. Conflict of interest statement: None. Authorship: Y.T.-B. wrote the initial drafts of this manuscript and conducted many statistical analyses. K.J.-S. is the Adventist Health Study-2 study research nutritionist. Over many years she was integrally involved in preparation of the nutritional data for analyses, including the dairy/Ca data and other dietary covariates used in this study. She closely reviewed the final manuscript. A.M. is a statistician/data manager who prepared the data files, with imputation of missing data. He also reviewed the final manuscript. G.E.F. performed the final analyses for this manuscript, and re-wrote segments of the manuscript. He also received funding for this work and conceived of the study. Ethics of human subject participation: This study was conducted according to the guidelines laid down in the Declaration of Helsinki and all procedures involving human subjects were approved by the Internal Review Board of Loma Linda University. Written informed consent was obtained from all subjects.

\section{Supplementary material}

To view supplementary material for this article, please visit https://doi.org/10.1017/S1368980017001422

\section{References}

1. Ferlay J, Soerjomataram I, Ervik M et al. (2013) GLOBOCAN 2012 v1.0, Cancer Incidence and Mortality Worldwide: IARC CancerBase No. 11. http://globocan.iarc.fr (accessed August 2014).

2. Siegel R, DeSantis C \& Jemal A (2014) Colorectal cancer statistics, 2014. CA Cancer J Clin 64, 104-117.

3. Armstrong B \& Doll R (1975) Environmental factors and cancer incidence and mortality in different countries, with special reference to dietary practices. Int J Cancer 15, 617631.

4. Austin GL, Adair LS, Galanko JA et al. (2007) A diet high in fruits and low in meats reduces the risk of colorectal adenomas. J Nutr 137, 999-1004.

5. Benito E, Cabeza E, Moreno B et al. (1993) Diet and colorectal adenomas: a case-control study in Majorca. Int J Cancer 55, 213-219.

6. Levi F, Pasche C, Lucchini F et al. (2001) Dietary fiber and the risk of colorectal cancer. Eur J Cancer 37, 2091-2096.

7. Mathew A, Peters U, Chatterjee N et al. (2003) Fat, fiber, fruits, vegetables, and risk of colorectal adenomas. Int $J$ Cancer 108, 287-282.

8. Mai V, Andrew F, Peters U et al. (2003) Dietary fibre and risk of colorectal cancer in the Breast Cancer Detection Demonstration Project (BCDDP) follow-up cohort. Int $J$ Epidemiol 32, 234-239.

9. Michels KB, Giovannucci E, Chan AT et al. (2006) Fruit and vegetable consumption and colorectal adenomas in the Nurses' Health Study. Cancer Res 66, 3942-3953.

10. Terry P, Giovannucci E, Michels KB et al. (2001) Fruit, vegetables, dietary fiber, and risk of colorectal cancer. J Natl Cancer Inst 93, 525-533.

11. Bingham SA, Day NE, Luben R et al. (2003) Dietary fibre in food and protection against colorectal cancer in the European Prospective Investigation into Cancer and Nutrition (EPIC): an observational study. Lancet 361, 1496-1501.

12. Hsing AW, McLaughlin JK, Chow W-H et al. (1998) Risk factors for colorectal cancer in a prospective study among US white men. Int J Cancer 77, 549-553. 
13. Singh P \& Fraser G (1998) Dietary risk factors for colon cancer in a low-risk population. Am J Epidemiol 148, 761-774.

14. Wakai K, Date C, Fukui M et al. (2007) Dietary fiber and risk of colorectal cancer in the Japan collaborative cohort study. Cancer Epidemiol Biomarkers Prev 16, 668-675.

15. National Dairy Council (2008) Dairy foods and cancer prevention. Dairy Council Digest 79, issue 1, 1-6.

16. Murphy N, Norat T, Ferrari P et al. (2013) Consumption of dairy products and colorectal cancer in the European Prospective Investigation into Cancer and Nutrition (EPIC). PLoS One 8, e72715.

17. Järvinen R, Knekt P, Hakulinen T et al. (2001) Prospective study on milk products, calcium and cancers of the colon and rectum. Eur J Clin Nutr 55, 1000-1007.

18. Kearney J, Giovannucci E, Rimm EB et al. (1996) Calcium, vitamin $\mathrm{D}$, and dairy foods and the occurrence of colon cancer in men. Am J Epidemiol 143, 907-917.

19. Cho E, Smith-Warner SA, Spiegelman D et al. (2004) Dairy foods, calcium, and colorectal cancer: a pooled analysis of 10 cohort studies. J Natl Cancer Inst 96, 1015-1022.

20. World Cancer Research Fund/American Institute for Cancer Research (2011) Continuous Update Project Report. Food, Nutrition, Physical Activity, and the Prevention of Colorectal Cancer. http://www.wcrf.org/int/research-wefund/continuous-update-project-findings-reports/colorectalbowel-cancer (accessed July 2017).

21. Tantamango-Bartley Y, Jaceldo-Siegl K, Fan J et al. (2013) Vegetarian dietary patterns and the incidence of cancer in a low-risk population. Cancer Epidemiol Biomarkers Prev 22, 286-294.

22. Butler TL, Fraser GE, Beeson WL et al. (2008) Cohort profile: the Adventist Health Study-2 (AHS-2). Int J Epidemiol 37, 260-265.

23. Jaceldo-Siegl K, Fan J, Sabaté J et al. (2011) Race-specific validation of food intake obtained from a comprehensive food frequency questionnaire: Adventist Health Study-2. Public Health Nutr 14, 1988-1997.

24. US Department of Agriculture, Agricultural Research Service (2011) USDA National Nutrient Database for Standard Reference, Release 21. https://www.ars.usda.gov/northeastarea/beltsville-md/beltsville-human-nutrition-research-center/ nutrient-data-laboratory/docs/sr21-home-page/ (accessed July 2017).

25. Jaceldo-Siegl K, Knutsen SF, Sabate J et al. (2010) Validation of nutrient intake using an FFQ and repeated $24 \mathrm{~h}$ recalls in black and white subjects of the Adventist Health Study-2 (AHS-2). Public Health Nutr 13, 812-819.

26. R Core Team (2015) R: A Language and Environment for Statistical Computing. Vienna: R Foundation for Statistical Computing.

27. Fraser G \& Yan R (2007) Guided multiple imputation of missing data: using a subsample to strengthen the missingat-random assumption. Epidemiology 18, 246-252.

28. Schafer JL (1997) Inference by data augmentation. In Analysis of Incomplete Multivariate Data, 1st ed., pp. 89-143 [D Cox, V Isham, N Keiding et al., editors]. London: Chapman \& Hall.

29. Schakel S, Sievert Y \& Buzzard I (1988) Sources of data for developing and maintaining a nutrient database. J Am Diet Assoc 88, 1268-1271.

30. American Cancer Society (2014) Colorectal Cancer Prevention and Early Detection. https://www.cancer.org/ cancer/colon-rectal-cancer/detection-diagnosis-staging/acsrecommendations.html (accessed July 2017).

31. Thomas D, Stram D \& Dwyer J (1993) Exposure measurement error: influence on exposure-disease. Relationships and methods of correction. Annu Rev Public Health 14, 69-93.
32. Fraser $\mathrm{G}$ (2003) A search for truth in dietary epidemiology. Am J Clin Nutr 78, 2 Suppl., 521S-525S.

33. Efron B \& Tibshirani $\mathrm{R}$ (1993) An Introduction to the Bootstrap. Monographs on Statistics and Applied Probability. New York: Chapman \& Hall.

34. McCullough ML, Robertson AS, Rodriguez C et al. (2003) Calcium, vitamin D, dairy products, and risk of colorectal cancer in the Cancer Prevention Study II Nutrition Cohort (United States). Cancer Causes Control 14, 1-12.

35. Larsson SC, Bergkvist L, Rutegard J et al. (2006) Calcium and dairy food intakes are inversely associated with colorectal cancer risk in the Cohort of Swedish Men. Am J Clin Nutr 83, 667-673.

36. Aune D, Lau R, Chan DS et al. (2012) Dairy products and colorectal cancer risk: a systematic review and metaanalysis of cohort studies. Ann Oncol 23, 37-45.

37. Pietinen P, Malila N, Virtanen M et al. (1999) Diet and risk of colorectal cancer in a cohort of Finnish men. Cancer Causes Control 10, 387-396.

38. Larsson SC, Bergkvist L \& Wolk A (2005) High-fat dairy food and conjugated linoleic acid intakes in relation to colorectal cancer incidence in the Swedish Mammography Cohort. Am J Clin Nutr 82, 894-900.

39. Ralston RA, Truby H, Palermo CE et al. (2014) Colorectal cancer and nonfermented milk, solid cheese, and fermented milk consumption: a systematic review and meta-analysis of prospective studies. Crit Rev Food Sci Nutr 54, 1167-1179.

40. Norat T \& Riboli E (2003) Dairy products and colorectal cancer. A review of possible mechanisms and epidemiological evidence. Eur J Clin Nutr 57, 1-17.

41. Ye Q, Zheng Y, Fan S et al. (2014) Lactoferrin deficiency promotes colitis-associated colorectal dysplasia in mice. PLoS One 9, e103298.

42. Fujita K, Matsuda E, Sekine K et al. (2004) Lactoferrin enhances Fas expression and apoptosis in the colon mucosa of azoxymethane-treated rats. Carcinogenesis 25, 19611966.

43. Celasco G, Moro L, Aiello C et al. (2014) Calcium butyrate: anti-inflammatory effect on experimental colitis in rats and antitumor properties. Biomed Rep 2, 559-563.

44. Meraz-Torres LS \& Hernandez-Sanchez H (2012) Conjugated linoleic acid in dairy products: a review. Am J Food Technol 7, 176-179.

45. Kruger C (2011) Generally Recognized as Safe (GRAS) Determination for Cow's Milk-Derived Lactoferrin as a Component of Cow's Milk-Based Infant Forumlas, Cow's Milk Products, and Chewing Gum. Bethesda, MD: Spherix Consulting, Inc.

46. Zimmer J, Lange B, Frick JS et al. (2012) A vegan or vegetarian diet substantially alters the human colonic faecal microbiota. Eur J Clin Nutr 66, 53-60.

47. Wu K, Willett WC, Fuchs CS et al. (2002) Calcium intake and risk of colon cancer in women and men.J Natl Cancer Inst 94, 437-446.

48. Terry P, Baron JA, Bergkvist L et al. (2002) Dietary calcium and vitamin D intake and risk of colorectal cancer: a prospective cohort study in women. Nutr Cancer $\mathbf{4 3}$, 39-46.

49. Zheng W, Anderson KE, Kushi LH et al. (1998) A prospective cohort study of intake of calcium, vitamin D, and other micronutrients in relation to incidence of rectal cancer among postmenopausal women. Cancer Epidemiol Biomarkers Prev 7, 221-225.

50. Lipkin M \& Newmark H (1995) Calcium and the prevention of colon cancer. J Cell Biochem Suppl 22, 65-73. 